OPEN ACCESS

Edited by:

Aldo Corsetti

Università di Teramo, Italy

Reviewed by:

Anton Hartmann,

Helmholtz Zentrum München (HZ),

Germany

Atte Von Wright,

University of Eastern Finland,

Joensuu, Finland

*Correspondence:

Zhihong Sun

sunzhihong78@163.com

Specialty section:

This article was submitted to

Food Microbiology

a section of the journal

Frontiers in Microbiology

Received: 01 September 2017 Accepted: 09 November 2017 Published: 23 November 2017

Citation:

Zhang W, Guo H, Cao C, Li L, Kwok L-Y, Zhang H and Sun Z (2017) Adaptation of Lactobacillus casei Zhang to Gentamycin Involves an

Alkaline Shock Protein.

Front. Microbiol. 8:2316. doi: 10.3389/fmicb.2017.02316

\section{Adaptation of Lactobacillus casei Zhang to Gentamycin Involves an Alkaline Shock Protein}

\author{
Wenyi Zhang, Huiling Guo, Chenxia Cao, Lina Li, Lai-Yu Kwok, Heping Zhang and \\ Zhihong Sun*
}

Key Laboratory of Dairy Biotechnology and Engineering, Ministry of Education, Key Laboratory of Dairy Products Processing, Ministry of Agriculture, Inner Mongolia Agricultural University, Hohhot, China

Lactobacillus (L. casei) Zhang is a koumiss-originated probiotic strain, which was used as a model in a long-term antibiotics-driven evolution experiment to reveal bacterial evolutionary dynamics; and we isolated gentamycin-resistant $L$. casei Zhang descendents. To decipher the gentamycin resistance mechanism, here we cultivated the parental $L$. casei Zhang and its descendent cells in an antibiotics-containing environment to compare their global protein expression profiles using the iTRAQ-based proteomic approach. A total of 72 proteins were significantly up-regulated $(>2.0$-fold, $P<0.05)$, whilst 32 proteins were significantly down-regulated $<-2.0$-fold, $P<0.05$ ) in the descendent line. The gentamycin-resistant descendent line showed elevated expression in some carbohydrates, amino acids, and purine metabolic pathways. Several stress-related proteins were also differentially expressed. Among them, one alkaline shock protein, asp23, was up-regulated most in the gentamycin-resistant strain (21.9-fold increase compared with the parental strain). The asp23 gene disruption mutant was significantly more sensitive to gentamycin compared with the wild type, suggesting an important role of this gene in developing the gentamycin-resistant phenotype in L. casei. Our report has described the adaptation of a probiotic strain that has acquired antibiotics resistance through long-term antibiotics exposure at the proteome level, and we revealed a novel mechanism of gentamycin resistance.

Keywords: Lactobacillus casei Zhang, proteomic analysis, gentamycin, alkaline shock protein, asp23

\section{INTRODUCTION}

Lactobacillus (L. casei) is the dominant species within the genus Lactobacillus found in koumiss, a naturally fermented dairy product (Wu et al., 2009a). Besides, it has been detected in corn silage, wine, pickle, human gastrointestinal tracts, blood, yogurt, and cheese (Cai et al., 2007; Bao et al., 2016). The wide ecological distribution of $L$. casei reflects its flexibility in metabolizing carbohydrates (Zhang et al., 2010), and thus it is commonly used in food industry. The species Lactobacillus casei has been used both as starter cultures and as food additives for improving food texture properties (Dantas et al., 2016). Some strains are considered as probiotics bacteria due to their beneficial effects, such as antibacterial, antioxidative, and immunomodulatory properties (Ya et al., 2008; Zhang et al., 2014b; Wang et al., 2016). Meanwhile, the increasing availability of the whole genome sequences of representative $L$. casei strains and genetic tools for creating recombinant Lactobacillus has largely facilitated genetic and functional studies, leading to 
remarkable progress in our understanding of the cell biology of these bacteria (Xu and Kong, 2013; Lu et al., 2016).

Antibiotic resistance of bacteria is an increasingly serious public health threat (Normark and Normark, 2002). This is especially critical for pathogenic bacteria that can rapidly become antibiotic-resistant in response to clinical application of antibiotics (Arias and Murray, 2015). It is unlikely that the probiotics used in food industry face a similar situation due to low exposure to antibiotics within the food matrix. Moreover, strict regulations must be followed by the food industry to avoid unnecessary use of antibiotics (Lara et al., 2006). The dairy industry even routinely monitors raw milk against antibiotics contamination because such contaminants would suppress or even kill lactic acid bacteria (LAB) and subsequently affect milk fermentation. The addition of bacteriocins or bacteriocinproducing bacteria has been explored as a method of food preservation, but it is not a wide-spread practice (Galvez et al., 2007). However, owing to the imminent global health concern of bacterial antibiotic resistance, the use of probiotics has been proposed as a valuable adjunct or even alternative to antibiotic therapy in clinical practice due to both their health-promoting properties and intrinsic bacteriocidal effects (Boyanova and Mitov, 2012; Reid, 2017). Several clinical trials supported the use of probiotics in the management of acute gastroenteritis and antibiotic-associated diarrhea (Katz, 2010). In these situations, probiotics may be directly exposed to antibiotics, sometimes even at a high concentration. Thus, it would be crucial to characterize the adaptation of probiotics to antibiotics stress.

Lactobacillus casei Zhang is a probiotic strain isolated from koumiss (Zhang et al., 2014a, 2015). We previously performed a long-term evolution experiment using $L$. casei Zhang as a model to reveal bacterial evolutionary dynamics under antibiotic stress; and we generated gentamycin-resistant L. casei Zhang descendents (Wang et al., 2017). During the experiment, the bacteria developed resistance to gentamycin gradually, and the accumulation of genome point mutation stopped shortly after the descendent bacteria reached the maximum bacterial fitness. To decipher the mechanism of the resistance phenotype, here we compared the global protein expression profiles between the parental $L$. casei Zhang and its descendent cells grown under antibiotic selection force using the iTRAQ-based proteomic approach. Furthermore, we validated the expression of one selected differentially expressed protein using parallel reaction monitoring (PRM), followed by disrupting the corresponding gene to verify its function in the development of gentamycinresistant phenotype of $L$. casei Zhang.

\section{MATERIALS AND METHODS}

\section{Bacterial Isolates and Culture Conditions}

Lactobacillus casei Zhang-G-1200 was isolated from a longterm laboratory-based evolution experiment performed in our laboratory (Wang et al., 2017). The strain Zhang-G-1200 exhibited a higher resistance to gentamycin compared with the parental cells (minimum inhibitory concentration, MIC, of $32 \mu \mathrm{g} / \mathrm{mL}$ for Zhang-G-1200 vs. $2 \mu \mathrm{g} / \mathrm{mL}$ for the parental strain). The acquisition of resistance was a result of multigenerational and stepwise increase during the prolonged cultivation under gentamycin stress. Growth curves of the 2 strains were constructed based on optical density (OD) measurement every $2 \mathrm{~h}$ along 30-h fermentation in LSM supplemented with $1 \mu \mathrm{g} / \mathrm{mL}$ gentamycin. Meanwhile, changes in $\mathrm{pH}$ and viable counts were determined. All analyses were performed in triplicate.

The bacterial strain, Escherichia (E. coli) DH5 $\alpha$, was used as a host for standard cloning procedures. It was propagated aerobically in Luria Bertani broth at $37^{\circ} \mathrm{C}$. Chloramphenicol $(10 \mu \mathrm{g} / \mathrm{ml}$ for both E. coli and L. casei Zhang-G-1200) and erythromycin ( 250 and $5 \mu \mathrm{g} / \mathrm{ml}$ for E. coli and L. casei Zhang-G1200 , respectively) were used for selecting genetically modified bacterial clones.

\section{Sample Preparation, Protein Digestion and iTRAQ Labeling}

Both the parental and L. casei Zhang-G-1200 cells were collected after $24 \mathrm{~h}$ of cultivation in LSM supplemented with $1 \mu \mathrm{g} / \mathrm{mL}$ gentamycin. Four biological replicates were prepared for each of the L. casei Zhang and the Zhang-G-1200 strains. Cells were harvested by centrifugation, followed by washing with phosphate buffered saline for 4 times. One milliliter of lysis buffer was added to each sample, followed by sonication on ice and centrifugation at $13,000 \mathrm{rpm}$ for $10 \mathrm{~min}$ at $4^{\circ} \mathrm{C}$. The protein concentration of sample supernatants was determined by using the bicinchoninic acid protein assay.

Hundred microgram protein was transferred to a new tube and adjusted to a protein concentration of $1 \mu \mathrm{g} / \mu \mathrm{L}$ with $100 \mathrm{mM}$ triethylammonium bicarbonate (TEAB). Five microliters of $200 \mathrm{mM}$ DTT were added and incubated at $55^{\circ} \mathrm{C}$ for $1 \mathrm{~h}$, then $5 \mu \mathrm{L}$ of $375 \mathrm{mM}$ iodoacetamide was added to the sample and incubated for $30 \mathrm{~min}$ at room temperature in the dark. For each sample, proteins were precipitated with ice-cold acetone before re-dissolving in $20 \mu \mathrm{L}$ TEAB. Proteins were then tryptically digested with sequencing grade modified trypsin (Promega, Madison, WI), and the resultant peptide mixture was labeled using reagents from the iTRAQ reagents kit.

\section{High pH Reverse Phase Separation by UPLC}

The peptide mixture was redissolved in buffer A (buffer A: $10 \mathrm{mM}$ ammonium formate in water, $\mathrm{pH} 10$, adjusted with ammonium hydroxide) and then fractionated by high $\mathrm{pH}$ separation using an Aquity UPLC system (Waters Corporation, Milford, MA) connected to a reverse phase column (BEH C18 column, 2.1 $\times 150 \mathrm{~mm}, 1.7 \mu \mathrm{m}, 300 \AA$, Waters Corporation, Milford, MA). High $\mathrm{pH}$ separation was done using a linear gradient, starting from $0 \%$ B to $45 \%$ B in $35 \mathrm{~min}$ (B: $10 \mathrm{mM}$ ammonium formate in $90 \%$ acetonitrile, $\mathrm{pH} 10.0$, adjusted with ammonium hydroxide). The column flow rate and temperature were maintained at 250 $\mu \mathrm{L} / \mathrm{min}$ and $45^{\circ} \mathrm{C}$, respectively. Sixteen fractions were collected, and each fraction was dried in a vacuum concentrator prior to the next step.

\section{Low pH Nano-HPLC-MS/MS Analysis}

The dried fractions were re-suspended in a solution made of solvent $\mathrm{C}$ and $\mathrm{D}$ (C: water with $0.1 \%$ formic acid; D: 
acetonitrile with $0.1 \%$ formic acid), separated by nano LC, and analyzed by on-line electrospray tandem mass spectrometry. The experiments were performed on a Nano Aquity UPLC system (Waters Corporation, Milford, MA) connected to a quadrupole-Orbitrap mass spectrometer (Q-Exactive) (Thermo Fisher Scientific, Bremen, Germany) equipped with an online nano-electrospray ion source. Eight microliters of peptide sample were loaded onto the trap column (Thermo Scientific Acclaim PepMap C18, $100 \mu \mathrm{m} \times 2 \mathrm{~cm}$ ), with a flow of $10 \mu \mathrm{l} / \mathrm{min}$ for $3 \mathrm{~min}$, and subsequently separated on an analytical column (Acclaim PepMap C18, $75 \mu \mathrm{m} \times 25 \mathrm{~cm}$ ) with a linear gradient, from $5 \% \mathrm{D}$ to $30 \% \mathrm{D}$ in $95 \mathrm{~min}$. The column was re-equilibrated to the initial conditions for $15 \mathrm{~min}$. The column flow rate and temperature were maintained at $300 \mathrm{~nL} / \mathrm{min}$ and $45^{\circ} \mathrm{C}$, respectively. An electrospray voltage of $2.0 \mathrm{kV}$ was used against the inlet of the mass spectrometer.

The Q Exactive Hybrid Quadrupole-Orbitrap Mass Spectrometer was operated in the data-dependent mode to switch automatically between MS and MS/MS acquisition. Survey full-scan MS spectra (m/z 350-1,600) were acquired with a mass resolution of $70 \mathrm{~K}$, followed by 15 sequential high-energycollisional-dissociation (HCD) MS/MS scans with a resolution of $17.5 \mathrm{~K}$. In all cases, one micro-scan was recorded using dynamic exclusion of $30 \mathrm{~s}$, with an MS/MS fixed first mass of 100 .

\section{Database Searching and Data Analysis}

Tandem mass spectra were extracted by the Proteome Discoverer software (Thermo Fisher Scientific, version 1.4.0.288). The mass profiles generated from all samples were searched with Mascot (Matrix Science, London, UK; version 2.3) against the NCBI database (Taxonomy: L. casei Zhang). We used the percolator algorithm $<1 \%$ to control peptide level false discovery rates. Only unique peptides were used for protein quantification and the normalization on protein median was applied to correct any experimental bias. The minimum number of proteins that must be observed was set to 1,000 . Students't-tests were performed with the software package $\mathrm{R}$, with $p<0.05$ considered statistically significant. A 2.0 -fold change was used as the threshold for selection of differentially regulated proteins. All regulated proteins were distributed over clusters of orthologous genes (COGs) and searched against the Kyoto Encyclopedia of Genes and Genomes database.

\section{Validation of Expression Level of an Alkaline Shock Protein by PRM}

Based on the results of proteomic analysis, 1 protein (the alkaline shock protein, coded by the gene LCAZH_0227) was selected for PRM analysis. This protein was most up-regulated among all differentially expressed proteins.

Experiments were performed on a Q Exactive mass spectrometer coupled with Easy-nLC1200. Peptide mixtures were separated by C18-reversed phase chromatography on an Easy column $(75 \mu \mathrm{m} \times 25 \mathrm{~cm})$, and the analytical separation was run for 90 min using a linear gradient of ACN/FA 2\%/0.1\% (Solvent A) and ACN/FA 80\%/0.1\% (Solvent B) at a flow rate of $300 \mathrm{~nL} / \mathrm{min}$. The gradient programme was run as follows: $5 \% \mathrm{~B}$ at $1 \mathrm{~min}$, ramping to $23 \% \mathrm{~B}$ at $41 \mathrm{~min}, 29 \% \mathrm{~B}$ at $51 \mathrm{~min}$, rapid ramping to $100 \% \mathrm{~B}$ over $8 \mathrm{~min}$ and holding $100 \% \mathrm{~B}$ for $6 \mathrm{~min}$ before returning to initial condition of $5 \% \mathrm{~B}$. The column was re-equilibrated to $5 \% \mathrm{~B}$ for $30 \mathrm{~min}$ after each run. All samples were analyzed using a multiplexed PRM method based on a scheduled inclusion list containing the target precursor ions representing the standard peptides. The full scan event was collected at $\mathrm{m} / \mathrm{z} 300-1300$, an Orbitrap resolution of 70,000, the automatic gain control target at $3 \mathrm{e} 6$, and the maximum fill time at $20 \mathrm{~ms}$. Every full scan was followed by 10 PRM scans at a resolution of 17,500 with an isolation window: $2.0 \mathrm{~m} / \mathrm{z}$, an AGC value of $5 \mathrm{e} 5$, the maximum fill time of $100 \mathrm{~ms}$, and a normalized collision energy of 28 in a higher-energy c-trap dissociation (HCD) cell.

PRM data analysis and data integration were performed with the Skyline Software. Routine assessment of instrument and chromatographic performance was done with a quality control (QC) standard consisting of all synthetic peptides, which was prepared at a concentration of $20 \mathrm{fmol} / \mu \mathrm{l}$ in $0.1 \%$ formic acid. Every sample was injected for three times, the peak areas of the target peptides were extracted using Skyline, peak peaking was manually checked and corrected in accordance to the retention time, transitions, mass accuracy, and MS/MS spectra. At least 3 transitions for each peptide were extracted from the PRM data. Peptides were quantified by summing the peak areas under curve (AUC) of each transition. Peptide abundance was normalized based on the total ion current (TIC) extracted from the full scan acquisition for each run. Proteins were quantified by summing the abundances of the selected peptides, and the accurate protein quantities had to match those of the synthetic standard peptides.

\section{Construction and Analysis of an Alkaline Shock Protein Gene Disruption Mutant}

The gene LCAZH_0227 (asp23) was selected for target disruption. Plasmids and primers used for gene disruption are listed in Table 1. The mutant line was constructed by using a crelox-based system originally developed by Lambert et al. (2007). Briefly, the upstream (amplified with the primers 0227upF and 0227upR,) and downstream (amplified with 0227downF and 0227downR) fragments of the LCAZH_0227 gene were PCR amplified from the genomic DNA of L. casei Zhang-G-1200. The fragments were cloned between the SalI-HF or PmeI and Ecol $53 \mathrm{KI}$ or BglII restriction sites of the suicide vector pNZ5319 to form the recombinant mutagenesis vector, pNZ5319-0227 Up-Down. To inactivate the LCAZH_0227 gene, pNZ53190227 Up-Down was introduced into L. casei Zhang-G-1200 by electroporation. Chloramphenicol-resistant transformants were selected and replica plated to check for an erythromycin-sensitive phenotype. Candidate double-crossover mutant clones were identified by PCR, and correct integration of the lox66-P32-catlox71 cassette into the genome was further verified by PCR using the primers $0227 \mathrm{upF}$ or catR and catF or 0227 downR. To excise the P32-cat selectable marker cassette, the cre expression plasmid pMSPcre was transformed into the 0227::lox66-P32-cat-lox71 gene replacement mutant. The Cre-mediated recombination and correct excision of the P32-cat cassette were checked by PCR using primers spanning the recombination loci $(0227 \mathrm{upF}$ and 
TABLE 1 | Strains, primers, and plasmids used for constructing the gene disruption mutant.

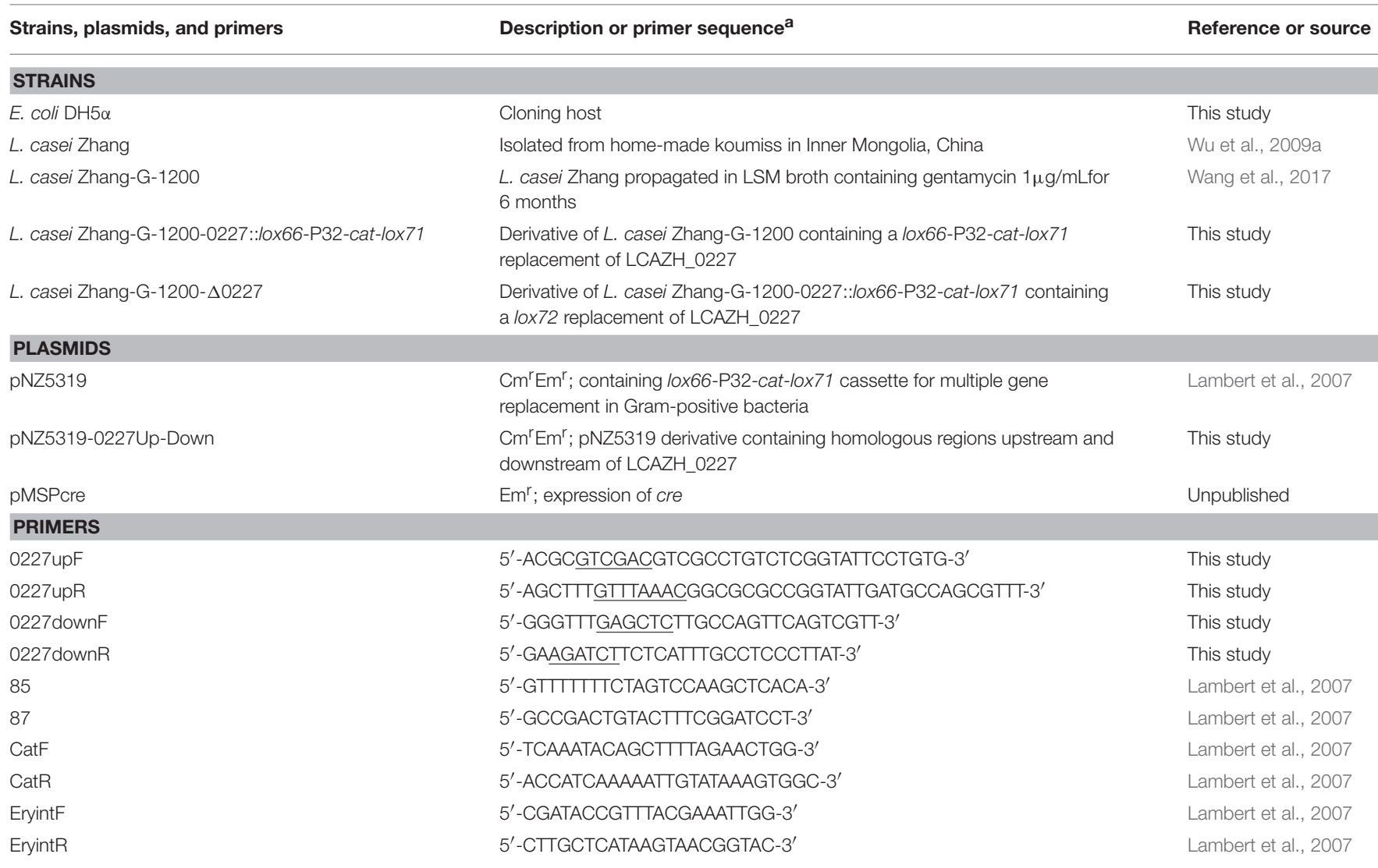

${ }^{a}$ The restriction sites in the primer sequences are underlined.

0227downR). The pMSPcre vector was cured from $L$. casei Zhang-G-1200 $\Delta 0227$ colonies by growth without erythromycin selection pressure. Additionally, PCR products were confirmed by sequencing when necessary.

The growth performance and gentamycin-resistant phenotype of the mutant strain was evaluated by viable counts, OD measurements, and MIC of gentamycin (Guo et al., 2017). Phenotypic differences between the wild-type and mutant strains were determined statistically by Student's $t$-test.

\section{RESULTS}

\section{Growth Performance of the Parental and L. Casei Zhang-G-1200 Strains}

The growth of the two bacterial lines in the presence of gentamycin was monitored. Growth curves were plotted based on viable counts, $\mathrm{pH}$ values, and $\mathrm{OD}$ values. As shown in Figures 1A-C, the growth performance of $L$. casei Zhang-G-1200 was completely different from that of the parental line. The viable counts of $L$. casei Zhang-G-1200 increased more rapidly than that of the parental line, reaching a maximum cell density of 9.05 $\times 10^{7} \mathrm{cfu} / \mathrm{mL}$ after $20 \mathrm{~h}$ (Figure 1A). Meanwhile, the $\mathrm{pH}$ of the LSM medium inoculated with $L$. casei Zhang-G-1200 dropped much faster than that of the parental line, indicating a higher fermentation rate (Figure 1B).

\section{Up-regulated L. Casei Zhang-G-1200 Proteins Grown in Gentamycin-Containing Medium}

A total of 72 proteins were significantly up-regulated $(>2.0$-folds, $P<0.05)$ in L. casei Zhang-G-1200 compared with the parental strain (Table 2). Most of these proteins could be assigned to one of the functional COGs categories (Figure 2), with 26.3\% of these proteins involved in carbohydrate transport and metabolism [G] and another $25.3 \%$ of them involved in amino acid transport and metabolism [E].

Over half (6 out of 10) of the up-regulated proteins were associated with the COGs category of carbohydrate transport and metabolism [G], which were the phosphotransferase system (PTS)-related components located within 3 different operons. According to the Transporter Classification Database (TCDB), 4 of these differentially regulated proteins belonged to the PTS Galactitol family, including the galacitol transporter subunits EIIA (LCAZH_2649), EIIB (LCAZH_2648), IIB (LCAZH_0604), and EIIC (LCAZH_0605), while the other 2 belonged to the PTS Lactose-N, N'-Diacetylchitobiose- $\beta$-glucoside family, namely the cellobiose-specific transporter subunits IIA (LCAZH_1771) and IIB (LCAZH_1772).

Another group of significantly up-regulated proteins was responsible for the uptake of oligopeptides (LCAZH_2023LCAZH_2026). This genomic cluster coded for the ATPase 

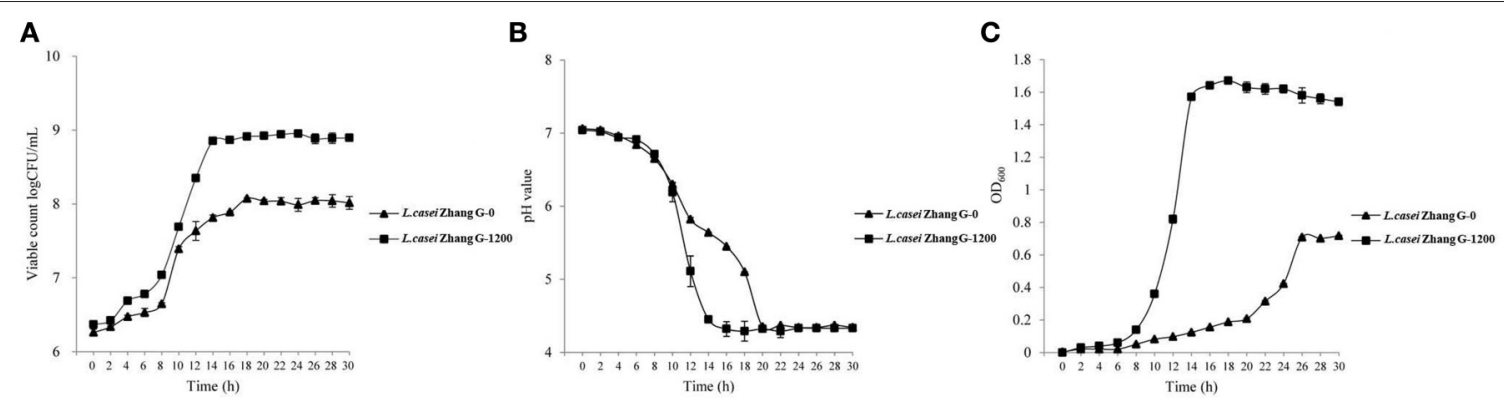

FIGURE 1 | Growth curves of the parental and L. casei Zhang-G-1200 strains based on viable counts (A), pH values (B) and OD values (C) in gentamycin-containing LSM.

and 2 permease components of a dipeptide/oligopeptide/nickel $\mathrm{ABC}$ transporter, as well as an oligopeptide $\mathrm{ABC}$ transporter periplasmic protein. Similar to the Opp system identified in other LAB genomes (Chen et al., 2015), another gene that coded for the ATP-binding subunit of the oligopeptide $\mathrm{ABC}$ transporter was found immediately downstream of the oligopeptide $\mathrm{ABC}$ transporter periplasmic protein of the genome of L. casei Zhang (Zhang et al., 2010). Besides, several other oligopeptide (LCAZH_0201, LCAZH_1596, LCAZH_1886, and LCAZH_2851) and amino acid (LCAZH_0500 and LCAZH_0418) transporter components, as well as subunits for de novo syntheses of tryptophan (LCAZH_0084) and glutamate (LCAZH_2518 and LCAZH_2519), were identified.

Other up-regulated proteins included those relating to purine biosynthesis (LCAZH_1739, LCAZH_1740, and LCAZH_1743) and stress response (LCAZH_1344 and LCAZH_0227). Notably, the alkaline shock protein (LCAZH_0227) was the most significantly up-regulated protein with a 21.9 -fold increase in its expression compared with the parental line, suggesting its important role in gentamycin adaptation.

\section{Down-regulated L. Casei Zhang-G-1200 Proteins Grown in Gentamycin-Containing Medium}

A total of 36 proteins were significantly down-regulated (<-2.0-folds, $P<0.05)$ in L. casei Zhang-G-1200 compared with the parental strain (Table 3). According to the COGs functional classification, $33.3 \%$ of these proteins were involved in posttranslational modification, protein turnover, chaperones metabolism [G] (Figure 2). Indeed, most of them were stress-responsive proteins, including the protease subunit of ATP-dependent Clp protease (LCAZH_0907), the molecular chaperones GrpE, GroEL, and HSP20-2 (LCAZH_1553, LCAZH_2207, and LCAZH_2811), the co-chaperonin GroES (LCAZH_2208), and the Clp protease/DnaK/DnaJ chaperone ATP-binding subunit (LCAZH_1753), while the others were mainly hypothetical proteins of unknown functions.

\section{Validation of Expression Level of an Alkaline Shock Protein by PRM}

The expression of the alkaline shock protein (LCAZH_0227) was confirmed by PRM analysis. Fortunately, 1 unique peptide
(FDDAVIAK) corresponding to this protein was found, which enabled the downstream quantification. As revealed by the analysis, the quantity of this alkaline shock protein was significantly higher in $L$. casei Zhang-G-1200 than the parental cells $(2.938 \pm 0.144 \mathrm{fmol} / \mu \mathrm{g}$ vs. $0.789 \pm 0.057 \mathrm{fmol} / \mu \mathrm{g})$. This result was consistent with the findings of the proteomic analysis.

\section{Partial Reversion of Gentamycin-Resistant Phenotype of asp23 Disruption Mutant}

The asp23 gene (LCAZH_0227) was selected as a target for mutational analysis because it was the most up-regulated protein in L. casei Zhang-G-1200 when the cells were grown in the presence of gentamycin. As shown in Figure 3, asp23 inactivation did not affect the growth of L. casei Zhang-G-1200 in LSM without antibiotics. However, the L. casei Zhang-G-1200 asp23 disruption mutant was significantly more sensitive to gentamycin with a relatively low MIC value of $8 \mu \mathrm{g} / \mathrm{mL}$ though still higher than that of the parental strain (vs. $2 \mu \mathrm{g} / \mathrm{mL}$ and $32 \mu \mathrm{g} / \mathrm{mL}$ for the parental strain and L. casei Zhang-G-1200, respectively). When the concentration of gentamycin increased to $16 \mu \mathrm{g} / \mathrm{mL}$, the wild type L. casei Zhang-G-1200 cells survived significantly better than the asp23 disruption mutant.

\section{DISCUSSION}

Probiotics that are used in food industry have low exposure to antibiotics normally. However, owing to their intrinsic antimicrobial property and desirable health-promoting effects, some scientists and clinicians have suggested using them to manage gastrointestinal disorders in clinical cases, when they may be directly exposed to antibiotics, sometimes even at a high concentration. Thus, it would be crucial to understand the evolutionary adaptation mechanisms of probiotics bacteria toward antibiotics as part of the risk assessment. In a previous long-term antibiotic-driven evolution experiment, we isolated the L. casei Zhang-G-1200 strain that exhibited elevated resistance to gentamycin compared with the parental line. The present study further characterized the mechanism of gentamycin resistance of this isolate using the iTRAQ-based proteomic approach. 
TABLE 2 | Up-regulated proteins of L. casei Zhang-G-1200 compared with its original strain in the presence of the gentamycin.

\begin{tabular}{|c|c|c|}
\hline Locus & Function & Fold change \\
\hline \multicolumn{3}{|c|}{ CARBOHYDRATE TRANSPORT AND METABOLISM } \\
\hline LCAZH_0394 & Hypothetical protein & 2.07 \\
\hline LCAZH_0403 & $\begin{array}{l}\text { PTS system mannose/fructose/N-acetylgalactosamine-specific transporter subunit } \\
\text { IIB }\end{array}$ & 2.23 \\
\hline LCAZH_0605 & PTS system galacitol transporter subunit ElIC & 2.22 \\
\hline LCAZH_1336 & Tagatose-6-phosphate kinase & 2.41 \\
\hline LCAZH_1768 & Beta-glucosidase/6-phospho-beta-glucosidase/beta-galactosidase & 2.13 \\
\hline LCAZH_1771 & PTS system cellobiose-specific transporter subunit IIA & 2.2 \\
\hline LCAZH_1772 & PTS system cellobiose-specific transporter subunit IIB & 2.61 \\
\hline LCAZH_2648 & PTS system galacitol transporter subunit ElIB & 5.79 \\
\hline LCAZH_2649 & PTS system galacitol transporter subunit EllA & 3.96 \\
\hline LCAZH_2653 & Trehalose-6-phosphate hydrolase & 4.66 \\
\hline LCAZH_2725 & Transaldolase & 4.56 \\
\hline LCAZH_2895 & PTS system mannitol-specific transporter subunit IIBC & 3.96 \\
\hline \multicolumn{3}{|c|}{ AMINO ACID TRANSPORT AND METABOLISM } \\
\hline LCAZH_0084 & Tryptophan synthase subunit alpha & 2.25 \\
\hline LCAZH_0107 & Tetrahydrodipicolinate $\mathrm{N}$-succinyltransferase & 2.25 \\
\hline LCAZH_0201 & Oligopeptide ABC transporter periplasmic protein & 2.04 \\
\hline LCAZH_0418 & Amino acid ABC transporter ATP-binding protein & 2.14 \\
\hline LCAZH_2024 & Dipeptide/oligopeptide/nickel ABC transporter permease & 2.45 \\
\hline LCAZH_2025 & Dipeptide/oligopeptide/nickel ABC transporter permease & 3.27 \\
\hline LCAZH_2026 & Oligopeptide ABC transporter periplasmic protein & 2.68 \\
\hline LCAZH_2111 & Homoserine dehydrogenase & 2.36 \\
\hline LCAZH_2302 & Aminopeptidase & 6.77 \\
\hline LCAZH_2518 & NADPH-dependent glutamate synthase subunit beta-like oxidoreductase & 3.52 \\
\hline LCAZH_2519 & Glutamate synthase domain 3 & 2.54 \\
\hline LCAZH_2851 & Polar amino acid ABC transporter ATPase & 2.23 \\
\hline \multicolumn{3}{|c|}{ ENERGY PRODUCTION AND CONVERSION } \\
\hline LCAZH_0188 & Acetate kinase & 2.17 \\
\hline LCAZH_1301 & $\begin{array}{l}\text { Acetoin/pyruvate dehydrogenase complex, E2 component, dihydrolipoamide } \\
\text { succinyltransferase }\end{array}$ & 2.05 \\
\hline LCAZH_1302 & $\begin{array}{l}\text { Acetoin/pyruvate dehydrogenase complex, E3 component, dihydrolipoamide } \\
\text { dehydrogenase }\end{array}$ & 2.13 \\
\hline LCAZH_1396 & Pyruvate-formate lyase & 3.75 \\
\hline LCAZH_2375 & Fumarase & 2.1 \\
\hline
\end{tabular}


TABLE 2 | Continued

\begin{tabular}{|c|c|c|}
\hline Locus & Function & Fold change \\
\hline \multicolumn{3}{|c|}{ NUCLEOTIDE TRANSPORT AND METABOLISM } \\
\hline LCAZH_1739 & Folate-dependent phosphoribosylglycinamide formyltransferase PurN & 2.5 \\
\hline LCAZH_1740 & Phosphoribosylaminoimidazole (AIR) synthetase & 2.23 \\
\hline LCAZH_1743 & $\begin{array}{l}\text { Phosphoribosylformylglycinamidine (FGAM) synthase, glutamine amidotransferase } \\
\text { domain }\end{array}$ & 2.21 \\
\hline \multicolumn{3}{|c|}{ CELL WALL/MEMBRANE/ENVELOPE BIOGENESIS } \\
\hline LCAZH_0447 & Conjugated bile salt hydrolase-like amidase & 5.04 \\
\hline \multicolumn{3}{|c|}{ TRANSCRIPTION } \\
\hline LCAZH_1410 & GNAT family acetyltransferase & 2.42 \\
\hline LCAZH_2210 & Transcriptional regulator & 3.18 \\
\hline \multicolumn{3}{|c|}{ COENZYME TRANSPORT AND METABOLISM } \\
\hline LCAZH_1463 & Lipoate-protein ligase A & 2.05 \\
\hline \multicolumn{3}{|c|}{ TRANSLATION, RIBOSOMAL STRUCTURE AND BIOGENESIS } \\
\hline LCAZH_1880 & Acetyltransferase & 3.78 \\
\hline \multicolumn{3}{|c|}{ POSTTRANSLATIONAL MODIFICATION, PROTEIN TURNOVER, CHAPERONES } \\
\hline LCAZH_0279 & ADP-ribosylglycohydrolase & 2.51 \\
\hline LCAZH_1344 & Chaperone ClpB & 3.68 \\
\hline LCAZH_1380 & Peptide methionine sulfoxide reductase & 2.23 \\
\hline LCAZH_1398 & Pyruvate-formate lyase-activating enzyme & 2.88 \\
\hline \multicolumn{3}{|c|}{ DEFENSE MECHANISMS } \\
\hline LCAZH_1217 & Multidrug ABC transporter ATPase & 6.01 \\
\hline \multicolumn{3}{|c|}{ GENERAL FUNCTION PREDICTION ONLY } \\
\hline LCAZH_0294 & Alpha/beta hydrolase & 2.64 \\
\hline LCAZH_0305 & NAD(FAD)-dependent dehydrogenase & 2.14 \\
\hline LCAZH_0638 & ABC transporter periplasmic protein & 2.06 \\
\hline LCAZH_0641 & ABC transporter permease & 2.3 \\
\hline LCAZH_0642 & ABC transporter ATPase & 2.15 \\
\hline LCAZH_1865 & Dinucleotide-binding enzyme & 2.05 \\
\hline LCAZH_2372 & Oxidoreductase & 2.53 \\
\hline LCAZH_2373 & Short-chain alcohol dehydrogenase & 3.71 \\
\hline \multicolumn{3}{|c|}{ FUNCTION UNKNOWN } \\
\hline LCAZH_0227 & Alkaline shock protein & 21.93 \\
\hline LCAZH_2030 & Hypothetical protein & 3.57 \\
\hline LCAZH_2301 & Putative integral membrane protein & 3.32 \\
\hline LCAZH_2056 & Hypothetical protein & 2.3 \\
\hline LCAZH_2222 & Hypothetical protein & 3.29 \\
\hline LCAZH_1464 & Hypothetical protein & 3.26 \\
\hline LCAZH_1898 & Hypothetical protein & 2.42 \\
\hline LCAZH_0186 & Hypothetical protein & 2.34 \\
\hline
\end{tabular}

Lactobacillus casei is a highly adaptable bacterium that can live on a wide range of niches; and it has a great capacity for choosing specific nutritional elements that enable its growth within any complex environments (Wang et al., 2012b). Like many other bacteria, it has developed sophisticated cellular mechanisms to regulate its nutritional responses. Carbon is one of the most important macronutrients for the bacterial cells; thus, its carbon metabolism and regulation have been studied in detail (Titgemeyer and Hillen, 2002). The strong ability L. casei in carbohydrate utilization relies very much on a rich array of PTSs present in its genome, ranking highest among all members within the Lactobacillus genus (Zhang et al., 2010). The current proteomic analysis revealed an apparent up-regulated expression of some PTS-related components in the gentamycin-resistant L. casei Zhang-G-1200 strain. The main function of PTSs is catalyzing sugar transport and phosphorylation (Zhang et al., 2013). The enhanced expression in L. casei Zhang-G-1200 may suggest an elevated cellular demand for utilizing different carbon substrates due to the presence of gentamycin.

Amino acid regulation is another important aspect required for sustaining growth of $L$. casei, especially for the late growth stages (Wang et al., 2012a). L. casei Zhang is able 


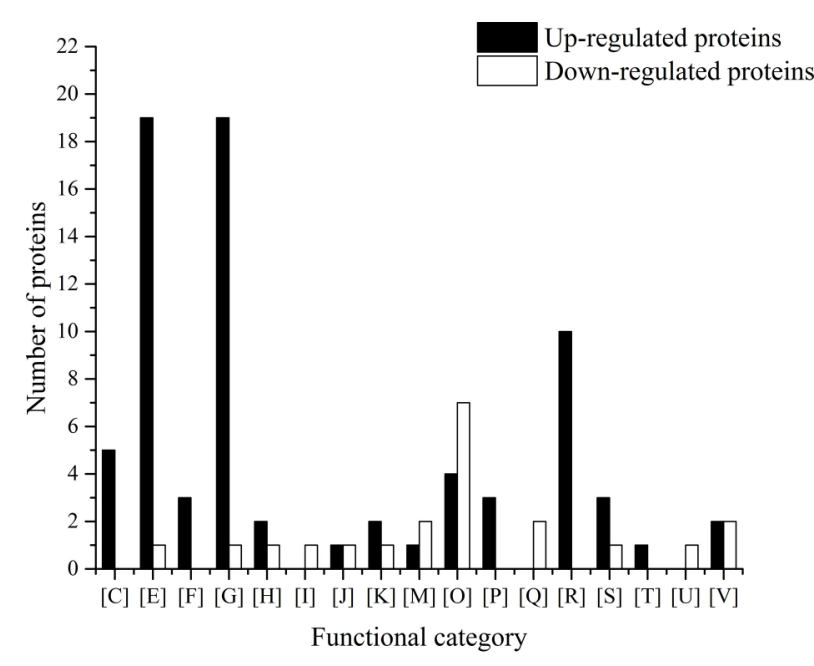

FIGURE 2 | Proteomic profiles of differentially expressed proteins of the parental L. casei Zhang G-0 and gentamycin-resistant L. casei Zhang-G-1200 strains. Clusters of orthologous groups (COG) functional categories: [C], Energy production and conversion; [E], Amino acid transport and metabolism; [F] Nucleotide transport and metabolism; [G], Carbohydrate transport and metabolism; $[\mathrm{H}]$, Coenzyme transport and metabolism; [l], Lipid transport and metabolism; [J], Translation, ribosomal structure and biogenesis; [K],

Transcription; [L], Replication, recombination and repair; [M], Cell wall/membrane/envelope biogenesis; [O], Posttranslational modification, protein turnover, chaperones; [P], Inorganic ion transport and metabolism; [Q], Secondary metabolites biosynthesis, transport and catabolism; [R], General function prediction only; [S], Function unknown; [T], Signal transduction mechanisms; [V], Defense mechanisms.

to synthesize most but the branch-chained amino acids; it is thus necessary for the bacterial cells to acquire the missing amino acids from the direct growth environment by proteolysis. L. casei Zhang possesses a well-developed proteolytic system (Wang et al., 2012b). In the gentamycincontaining environment, some of the key proteins involving in tryptophan and glutamate synthesis were found to be upregulated, e.g., the tryptophan synthase alpha subunit and the glutamate synthase subunits. The tryptophan synthase alpha subunit functions to convert indole-3-glycerolphosphate into indole, the terminal step of tryptophan biosynthesis (Lim et al., 1991), while the glutamate synthase (large subunit) and NADPHdependent glutamate synthase (small subunit) together catalyze the transamidation of the amide group from glutamine to 2oxoglutarate to form glutamate (Stannek et al., 2015). Moreover, there were several up-regulated amino acid transporters and transporter-associated components, which might facilitate the intake of dipeptide/oligopeptides from the growth medium. The simultaneous up-regulation of multiple transporters for dipeptide/oligopeptides may suggest a need for L. casei Zhang-G1200 to assimilate amino acids more efficiently under gentamycin stress.

Purine nucleotides are substrates for RNA and DNA synthesis; and they are essential for the growth of some LAB species (Wang et al., 2012a). Here, we also observed the up-regulation of some purine biosynthesis-related proteins. Among these proteins, the folate-dependent phosphoribosylglycinamide formyltransferase catalyzes the steps whereby the formyl derivatives of tetrahydrofolic acid are donated to the precursors of inosinic acid during its biosynthesis (Hartman and Buchanan, 1959); and the phosphoribosylformylglycinamidine synthase converts glycinamide ribotide into glycinamidine ribotide (Melnick and Buchanan, 1957). As in some other bacteria, the genome of $L$. casei Zhang contains a typical gene cluster for de novo purine biosynthesis, namely PurCDFHKLM, consisting of 12 distinct genes. The bacterial purine nucleotide synthesis is a 10-step pathway that produces inosinic acid from 5-phosphoribosyl 1-pyrophosphate (Ebbole and Zalkin, 1987). Since the culture medium used in the present study lacked purine compounds, the purine nucleotides must be obtained from the bacterial de novo biosynthesis, which was reflected by the up-regulation of these proteins.

Another group of differentially expressed proteins was the stress-related proteins. The molecular chaperones, such as GroEL and GroES, play a central role in the control of general stress responses (Lemos and Burne, 2002). The GroEL and several other chaperones of $L$. casei have been shown to be up-regulated in response to acid and bile stress ( $\mathrm{Wu}$ et al., 2009b, 2010). Here, we observed a general reduction in expression of these proteins in the L. casei Zhang-G-1200 strain compared with its parental line under gentamycin stress except for the Clp protein and an alkaline shock protein (encoded by LCAZH_0227, the asp2 gene). These simply indicate that Zhang-G-1200 coped better in the antibiotics-containing environment due to its long-term adaptation to the drug. This was also reflected by a better growth performance of the adapted strain than its parental line in the presence of gentamycin.

It is interesting to note the high expression of asp23 in L. casei Zhang-G-1200 (21.9-fold increase compared with the parental strain). The activation of this gene is normally associated with alkaline shock conditions (Kuroda et al., 1995). To investigate its role in the gentamycin-resistant phenotype in the adapted strain, we created an asp23 disruption mutant. The gene disruption mutant appeared to be significantly more sensitive to gentamycin compared with the wild type L. casei Zhang-G-1200, though it was still more resistant than the parental L. casei Zhang, suggesting that this alkaline shock protein was partially responsible for the resistant phenotype. The bactericidal action of gentamycin resembles that of a ribosome modulation factor via the irreversible binding of the bacterial $30 \mathrm{~S}$ ribosomal subunit and hence interrupting protein synthesis. Some stress-responsive proteins are also known to serve as ribosome modulation factors (Niven and EI-Sharoud, 2008). For example, the E. coli $R b f A$ is a cold shock protein that regulates cold shock response by binding to the $30 \mathrm{~S}$ ribosomal binding factor; its absence promotes cold shock responses (Jones and Inouye, 1996). Although the exact cellular role of asp23 remains to be determined, it is tempting to speculate that it acts as a modulator of similar nature and competes with gentamycin. However, the facts that the gentamycin-resistant phenotype of Zhang-G-1200 was developed stepwise along the 
TABLE 3 | Down-regulated proteins of L. casei Zhang-G-1200 compared with its original strain in the presence of the gentamycin.

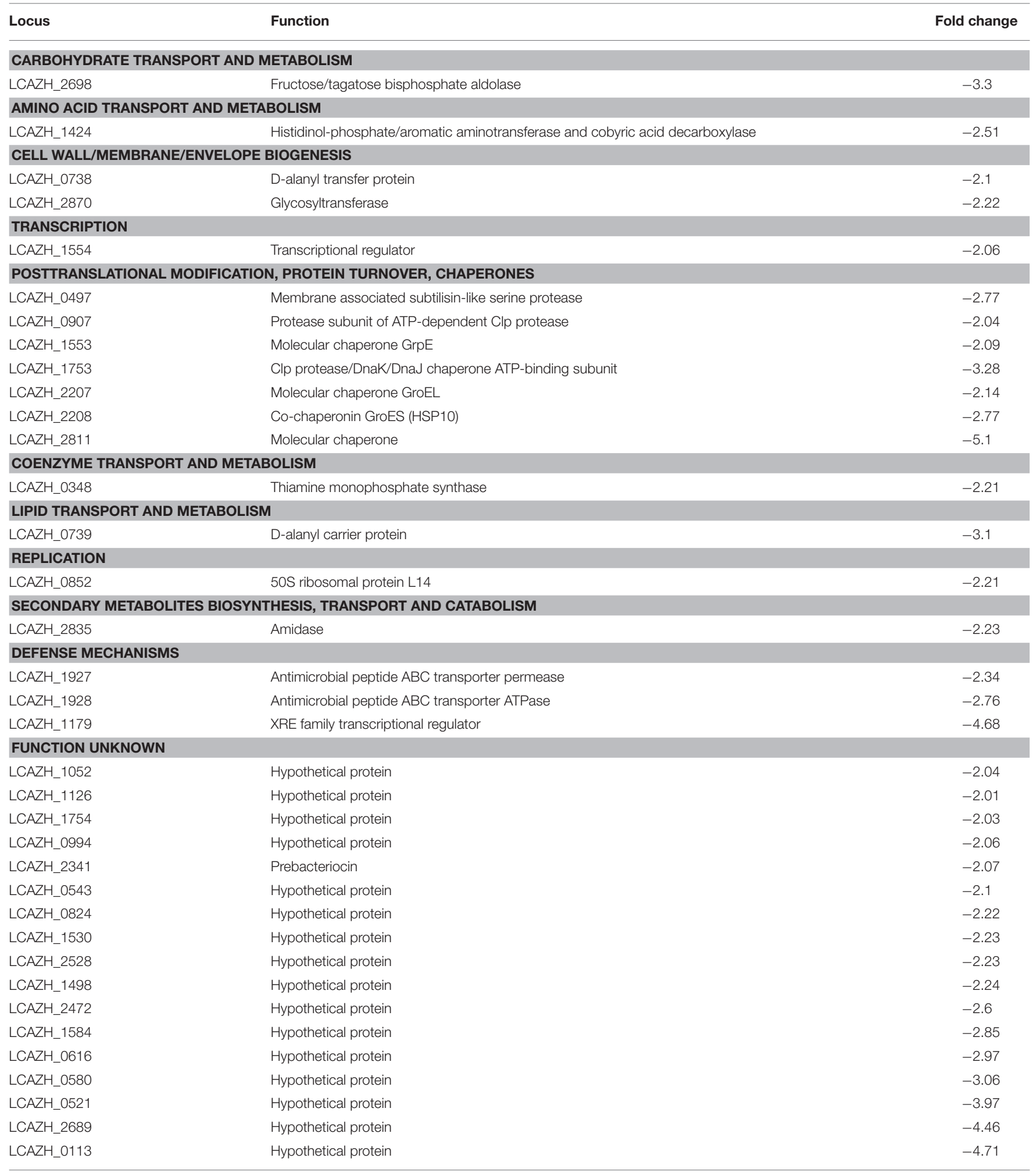

long-term cultivation and only partial reversion of gentamycinresistant phenotype was observed in the asp23 disruption mutant together suggest that the resistant phenotype was not solely caused by the asp23 gene but possibly along with certain secondary pleiotrophic mutations accumulated in the bacterial genome. To our knowledge, this work reports for the first time the involvement of alkaline shock proteins in gentamycin resistance. 

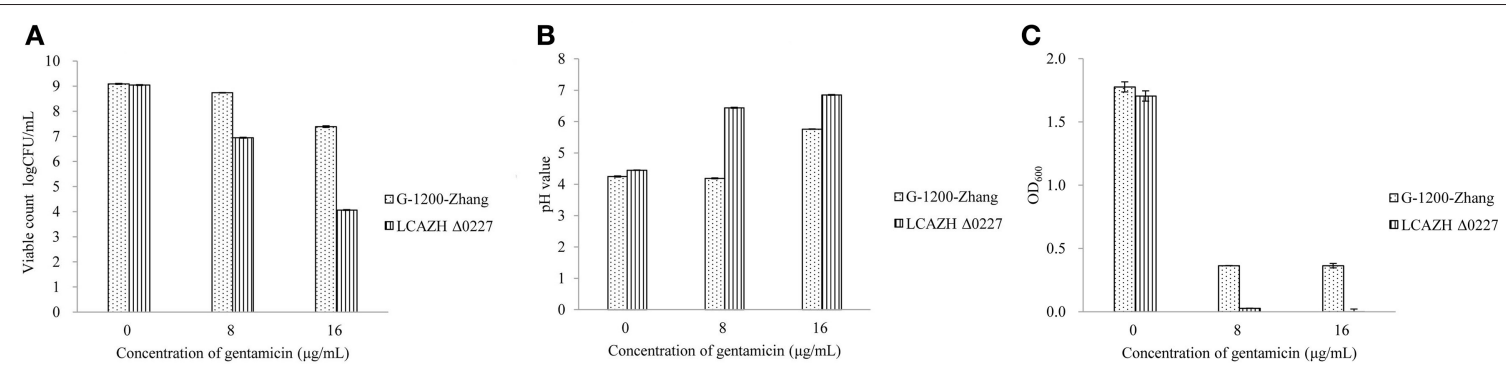

FIGURE 3 | The viable counts (A), pH values (B) and OD values (C) of the wild-type and mutant strains at the time of observing the minimum inhibitory concentration (MIC) by witness.

\section{CONCLUSION}

Several clinical trials supported the use of probiotics in the management of acute gastroenteritis and antibiotic-associated diarrhea, which elevates the chance of probiotics exposure to antibiotics. The present study characterized the mechanism of adaptive gentamycin resistance in a probiotic $L$. casei strain. We observed up-regulation in some carbohydrate, amino acid, and purine metabolic pathways in the resistant strain, which may function to support the bacterial growth under gentamycin stress. Meanwhile, some stress-related proteins were differentially expressed, particularly an alkaline shock protein encoded by the asp 23 gene. The disruption of asp 23 gene partially reversed the gentamycin-resistant phenotype, suggesting that this gene was involved in the resistance development although other accumulated mutations might play a role too. Normally, comparative proteomics should be performed in conditions, where the growth rates, cell densities and other culture parameters are as identical as possible between the strains that are compared. As the two strains studied here showed

\section{REFERENCES}

Arias, C. A., and Murray, B. E. (2015). A new antibiotic and the evolution of resistance. New Engl. J. Med. 372, 1168-1170. doi: 10.1056/NEJMcibr1500292

Bao, Q., Song, Y., Xu, H., Yu, J., Zhang, W., Menghe, B., et al. (2016). Multilocus sequence typing of Lactobacillus casei isolates from naturally fermented foods in China and Mongolia. J. Dairy Sci. 99, 5202-5213. doi: $10.3168 /$ jds.2016-10857

Boyanova, L., and Mitov, I. (2012). Coadministration of probiotics with antibiotics: why, when and for how long? Expert Rev. Anti Infect. Ther. 10, 407-409. doi: $10.1586 /$ eri.12.24

Cai, H., Rodriguez, B. T., Zhang, W., Broadbent, J. R., and Steele, J. L. (2007). Genotypic and phenotypic characterization of Lactobacillus casei strains isolated from different ecological niches suggests frequent recombination and niche specificity. Microbiology 153(Pt 8), 2655-2665. doi: 10.1099/mic. $0.2007 / 006452-0$

Chen, Y., Zhang, W., Sun, Z., Meng, B., and Zhang, H. (2015). Complete genome sequence of Lactobacillus helveticus $\mathrm{H} 9$, a probiotic strain originated from kurut. J. Biotechnol. 194, 37-38. doi: 10.1016/j.jbiotec.2014. 11.038

Dantas, A. B., Jesus, V. F., Silva, R., Almada, C. N., Esmerino, E. A., Cappato, L. P., et al. (2016). Manufacture of probiotic minas frescal cheese with Lactobacillus casei Zhang. J. Dairy Sci. 99, 18-30. doi: 10.3168/jds.2015-9880 different growth performance in the presence of gentamycin, some changes in the proteome could result from the different physiological status between cultures and not directly from a specific differential response to gentamycin.

\section{AUTHOR CONTRIBUTIONS}

$\mathrm{WZ}, \mathrm{ZS}$, and $\mathrm{HZ}$ designed the study. WZ, L-YK, and ZS wrote the manuscript. ZS, HG, CC, LL, and L-YK performed experiments. WZ and ZS analyzed data. All authors reviewed the manuscript.

\section{ACKNOWLEDGMENTS}

This research was supported by the National Natural Science Foundation of China (Grant Nos. 31571815 and 31622043) and the Program for Young Talents of Science and Technology in Universities of Inner Mongolia Autonomous Region (NJYT-17B05). We would like to thank Prof. Jian Kong for providing the plasmid pMSPcre.

Ebbole, D. J., and Zalkin, H. (1987). Cloning and characterization of a 12 gene cluster from Bacillus subtilis encoding nine enzymes for de novo purine nucleotide synthesis. J. Biol. Chem. 262, 8274-8287.

Galvez, A., Abriouel, H., Lopez, R. L., and Ben Omar, N. (2007). Bacteriocinbased strategies for food biopreservation. Int. J. Food Microbiol. 120, 51-70. doi: 10.1016/j.ijfoodmicro.2007.06.001

Guo, H., Pan, L., Li, L., Lu, J., Kwok, L., Menghe, B., et al. (2017). Characterization of antibiotic resistance genes from Lactobacillus isolated from traditional dairy products. J. Food Sci. 82, 724-730. doi: 10.1111/1750-3841.13645

Hartman, S. C., and Buchanan, J. M. (1959). Biosynthesis of the purines. XXVI. The identification of the formyl donors of the transformylation reactions. $J$. Biol. Chem. 234, 1812-1816.

Jones, P. G., and Inouye, M. (1996). RbfA, a 30 S ribosomal binding factor, is a coldshock protein whose absence triggers the cold-shock response. Mol. Microbiol. 21, 1207-1218. doi: 10.1111/j.1365-2958.1996.tb02582.x

Katz, J. (2010). Should probiotics be routine therapy for the prevention of antibiotic-associated diarrhea? J. Clin. Gastroenterol. 44, 83-84. doi: 10.1097/MCG.0b013e3181bdf010

Kuroda, M., Ohta, T., and Hayashi, H. (1995). Isolation and the gene cloning of an alkaline shock protein in methicillin resistant Staphylococcus aureus. Biochem. Biophys. Res. Commun. 207, 978-984. doi: 10.1006/bbrc.1995.1281

Lambert, J. M., Bongers, R. S., and Kleerebezem, M. (2007). Cre-loxbased system for multiple gene deletions and selectable-marker removal 
in Lactobacillus plantarum. Appl. Environ. Microbiol. 73, 1126-1135. doi: 10.1128/AEM.01473-06

Lara, F. J., Garcia-Campana, A. M., Ales-Barrero, F., Bosque-Sendra, J. M., and Garcia-Ayuso, L. E. (2006). Multiresidue method for the determination of quinolone antibiotics in bovine raw milk by capillary electrophoresis-tandem mass spectrometry. Anal. Chem. 78, 7665-7673. doi: 10.1021/ac061006v

Lemos, J. A., and Burne, R. A. (2002). Regulation and physiological significance of ClpC and ClpP in Streptococcus mutans. J. Bacteriol. 184, 6357-6366. doi: 10.1128/JB.184.22.6357-6366.2002

Lim, W. K., Shin, H. J., Milton, D. L., and Hardman, J. K. (1991). Relative activities and stabilities of mutant Escherichia coli tryptophan synthase alpha subunits. J. Bacteriol. 173, 1886-1893. doi: 10.1128/jb.173.6.1886-1893.1991

Lu, W. W., Wang, T., Wang, Y., Xin, M., and Kong, J. (2016). A food-grade fimbrial adhesin FaeG expression system in Lactococcus lactis and Lactobacillus casei. Can. J. Microbiol. 62, 241-248. doi: 10.1139/cjm-2015-0596

Melnick, I., and Buchanan, J. M. (1957). Biosynthesis of the purines. XIV. Conversion of (alpha-N-formyl) glycinamide ribotide to (alpha-N-formyl) glycinamidine ribotide; purification and requirements of the enzyme system. J. Biol. Chem. 225, 157-162.

Niven, G. W., and EI-Sharoud, W.M. (2008). "Ribosome modulation factor," in Bacterial Physiology, ed W. M. EI-Sharoud (Berlin; Heidelberg: Springer), 293-311.

Normark, B. H., and Normark, S. (2002). Evolution and spread of antibiotic resistance. J. Intern. Med. 252, 91-106. doi: 10.1046/j.1365-2796.2002.01026.x

Reid, G. (2017). Probiotic use in an infectious disease setting. Expert Rev. Anti Infect. Ther. 15, 449-455. doi: 10.1080/14787210.2017.1300061

Stannek, L., Thiele, M. J., Ischebeck, T., Gunka, K., Hammer, E., Volker, U., et al. (2015). Evidence for synergistic control of glutamate biosynthesis by glutamate dehydrogenases and glutamate in Bacillus subtilis. Environ. Microbiol. 17, 3379-3390. doi: 10.1111/1462-2920.12813

Titgemeyer, F., and Hillen, W. (2002). Global control of sugar metabolism: a gram-positive solution. Antonie Van Leeuwenhoek 82, 59-71. doi: 10.1023/A:1020628909429

Wang, J. C., Zhang, W. Y., Zhong, Z., Wei, A. B., Bao, Q. H., Zhang, Y., et al. (2012a). Gene expression profile of probiotic Lactobacillus casei Zhang during the late stage of milk fermentation. Food Control 25, 321-327. doi: 10.1016/j.foodcont.2011.10.036

Wang, J. C., Zhang, W. Y., Zhong, Z., Wei, A. B., Bao, Q. H., Zhang, Y., et al. (2012b). Transcriptome analysis of probiotic Lactobacillus casei Zhang during fermentation in soymilk. J. Ind. Microbiol. Biotechnol. 39, 191-206. doi: 10.1007/s10295-011-1015-7

Wang, J., Dong, X., Shao, Y., Guo, H., Pan, L., Hui, W., et al. (2017). Genome adaptive evolution of Lactobacillus casei under long-term antibiotic selection pressures. BMC Genomics 18:320. doi: 10.1186/s12864-017-3710-X

Wang, Y., Xie, J., Li, Y., Dong, S., Liu, H., Chen, J., et al. (2016). Probiotic Lactobacillus casei Zhang reduces pro-inflammatory cytokine production and hepatic inflammation in a rat model of acute liver failure. Eur. J. Nutr. 55, 821-831. doi: 10.1007/s00394-015-0904-3
Wu, R., Sun, Z., Wu, J., Meng, H., and Zhang, H. (2010). Effect of bile salts stress on protein synthesis of Lactobacillus casei Zhang revealed by 2-dimensional gel electrophoresis. J. Dairy Sci. 93, 3858-3868. doi: 10.3168/jds.2009-2967

Wu, R., Wang, L., Wang, J., Li, H., Menghe, B., Wu, J., et al. (2009a). Isolation and preliminary probiotic selection of lactobacilli from koumiss in Inner Mongolia. J. Basic Microbiol. 49, 318-326. doi: 10.1002/jobm.200800047

Wu, R., Wang, W., Yu, D., Zhang, W., Li, Y., Sun, Z., et al. (2009b). Proteomics analysis of Lactobacillus casei Zhang, a new probiotic bacterium isolated from traditional home-made koumiss in Inner Mongolia of China. Mol. Cell Proteomics 8, 2321-2338. doi: 10.1074/mcp.M800483MCP200

Xu, Y., and Kong, J. (2013). Construction and potential application of controlled autolytic systems for Lactobacillus casei in cheese manufacture. J. Food Prot. 76, 1187-1193. doi: 10.4315/0362-028X.JFP-12-307

Ya, T., Zhang, Q., Chu, F., Merritt, J., Bilige, M., Sun, T., et al. (2008). Immunological evaluation of Lactobacillus casei Zhang: a newly isolated strain from koumiss in Inner Mongolia, China. BMC Immunol. 9:68. doi: 10.1186/1471-2172-9-68

Zhang, J., Wang, L., Guo, Z., Sun, Z., Gesudu, Q., Kwok, L., et al. (2014a). 454 pyrosequencing reveals changes in the faecal microbiota of adults consuming Lactobacillus casei Zhang. FEMS Microbiol. Ecol. 88, 612-622. doi: 10.1111/1574-6941.12328

Zhang, W., Sun, Z., Menghe, B., and Zhang, H. (2015). Short communication: single molecule, real-time sequencing technology revealed species- and strainspecific methylation patterns of 2 Lactobacillus strains. J. Dairy Sci. 98, 3020-3024. doi: 10.3168/jds.2014-9272

Zhang, W. Y., Sun, Z. H., Wu, R. N., Meng, H., and Zhang, H. P. (2013). "Comparative genome analysis of a new probiotic strain Lactobacillus casei Zhang," Genomics II-Bacteria, Viruses and Metabolic Pathways (Brisbane: Iconcept Press), 276-296.

Zhang, W., Yu, D., Sun, Z., Wu, R., Chen, X., Chen, W., et al. (2010). Complete genome sequence of Lactobacillus casei Zhang, a new probiotic strain isolated from traditional homemade koumiss in inner Mongolia, China. J. Bacteriol. 192, 5268-5269. doi: 10.1128/JB.00802-10

Zhang, Y., Guo, X., Guo, J., He, Q., Li, H., Song, Y., et al. (2014b). Lactobacillus casei reduces susceptibility to type 2 diabetes via microbiota-mediated body chloride ion influx. Sci. Rep. 4:5654. doi: 10.1038/srep05654

Conflict of Interest Statement: The authors declare that the research was conducted in the absence of any commercial or financial relationships that could be construed as a potential conflict of interest.

Copyright (C) 2017 Zhang, Guo, Cao, Li, Kwok, Zhang and Sun. This is an open-access article distributed under the terms of the Creative Commons Attribution License (CC $B Y)$. The use, distribution or reproduction in other forums is permitted, provided the original author(s) or licensor are credited and that the original publication in this journal is cited, in accordance with accepted academic practice. No use, distribution or reproduction is permitted which does not comply with these terms. 